[4] K. Sarabandi, "Scattering from variable resistive and impedance sheets," J. Electromagn. Waves Appl, vol. 4, pp. 865-891, 1990.

[5] R. R. DeLyser and E. F. Kuester, "Homogenization analysis of electromagnetic strip gratings," J. Electromagn. Waves Appl,, vol. 5, no. 11, pp. 1217-1236, 1991.

[6] R. R. DeLyser, "Use of equivalent boundary conditions for the solution of a class of strip grating structures," IEEE Trans. Antennas Propagat., vol. 41, no. 1, pp. 103-105, Jan. 1993.

[7] M. Ando and M. Murota, "Reflection and transmission coefficients of a thin strip grating on a dielectric sheet," Trans. IECE, vol. E69, no. 11, Nov. 1986.

[8] A. Ishimaru, Electromagnetic Wave Propagation, Radiation, and Scattering. Englewood Cliffs, NJ: Prentice, 1991, pp. 190-194.

[9] J. L. Volakis, Y. C. Lin, and H. Anastasiou, "TE characterization of resistive strip gratings on a dielectric slab using a single edge-mode expansion," in Proc. 1993 IEEE AP-S Int. Symp. Digest, Ann Arbor MI, vol. 1, pp. 90-93.

[10] J. H. Richmond, "On the edge mode in the theory of TM scattering by a strip or strip grating," IEEE Trans. Antennas Propagat., vol. AP-28, pp. 883-887, Nov. 1980.

[11] D. R. Wilton and S. Govind, "Incorporation of edge conditions in moment method solutions," IEEE Trans. Antennas Propagat., vol. AP25, pp. 845-850, Nov. 1977.

[12] R. C. Hall and R. Mittra, "Scattering from a periodic array of resistive strips," IEEE Trans. Antennas Propagat., vol. AP-33, pp. 1009-1011, Sept. 1983.

[13] T. K. Wu, "Fast convergent integral equation solution of strip gratings on dielectic substrate," IEEE Trans. Antennas Propagat., vol. AP-35, no. 2, Feb. 1987.

[14] F. B. Gross and W. J. Brown, "New frequency dependent edge mode current density appoximations for TM scattering from a conducting strip grating," IEEE Trans. Antennas Propagat., vol. 41, pp. 1302-1307, Sept 1993.

[15] T. P. Silzer, "A new frequency dependent current density as applied to an infinite strip grating with transverse electric incidence," M.S. thesis, Florida State Univ., Fall 1992.

[16] R. Mittra, T. Itoh, and Ti-Shu Li, "Analytical and numerical studie of the relative convergence phenomenon arising in the solution of an integral equation by the moment method," IEEE Trans. Microwave Theory Tech., vol. MTT-18, pp. 627-632, Sept. 1970.

[17] T. Itoh and R. Mittra, "Relative convergence phenomena arising in the solution of diffraction from strip gratings on a dielectric slab," IEEE Proc. Lett., pp. 1363-1365, Sept. 1971

\section{Application of Conjugate Gradient Method for Static Problems Involving Conductors of Arbitrary Shape}

V. V. Sanjaynath, N. Balakrishnan, and G. R. Nagabhushana

Abstract-In this paper, two implementations of the Conjugate Gradient Method (CGM) for the solution of problems in electrostatics involving conductors of arbitrary shapes have been discussed. The first method uses a least squares approximation for the computation of the pertinent integral operator and is referred to as LSD. A second implementation, referred to as Point Matching Discretisation (PMD) effects considerable saving in the computer time since it uses the midpoint rule for the integral arising in LSD. Both these techniques require $O(N)$ storage, where $N$ is the number of patches used to model the conductor. Further, a matrix interpretation of the present formulation has been derived. This has facilitated the comparison of the techniques described in this paper with the well known Method of Moments (MoM) formulation and has led to better understanding of the convergence of the results. Using illustrative examples of canonical (square and circular discs) and arbitrary shape ( a pyramid mounted on a cube), the convergence of and the computer time for both the implementations have been investigated. It has been shown that both the techniques yielded monotonically convergent results for all the examples considered and that the LSD offers better estimate of the capacitance than PMD with lower number of patches.

\section{INTRODUCTION}

Computation of electrostatic fields involving arbitrary shaped conductors are of practical interest in electrical engineering. In these problems, one is usually interested in finding the charge distribution over the surface and the capacitance of the object. Since very few geometries yield analytical solutions, numerical approximations are of great use. One of the most popular methods for the numerical solution of such problems is the Method of Moments (MoM) [1]- [5]. One of the disadvantages of this approach is that they require the explicit storage of a dense matrix, which can be expensive even with today's mainframes. Another disadvantage is the problem of convergence which is discussed at length in the literature [4], [6]-[10]. It has been shown that a monotonic convergence in the solution can be achieved only under very stringent conditions, which are difficult to realize for complex problems [6].

In an attempt to address the above disadvantages of the conventional MoM, the Conjugate Gradient Method (CGM) has recently been suggested as an alternative tool for solving electromagnetic problems [11]-[13]. CGM has since then been successfully applied to a number of radiation and scattering problems. However, it has been convincingly argued [14] that almost all implementations of the conjugate gradient method are more or less equivalent to a moment method solution and hence the question of convergence is left unanswered. In spite of this, CGM has been quite popular because of the $O(N)$ memory requirement.

However, there have been only very few attempts to apply CGM to electrostatic problems involving conducting surfaces. Sarkar and Rao [15] applied the method of steepest descent to calculate the charge distribution over conducting surfaces. They applied the method to the matrix equation generated by a moment method discretisation as described in [3]. It may be noted that, the method of steepest descent

Manuscript received December 18, 1992; revised November 17, 1993

V. V. Sanjaynath and G. R. Nagabhushana are with the Department of High Voltage Engineering, Indian Institute of Science, Bangalore, India.

N. Balakrishnan is with the Department of Aerospace Engineering, Indian Institute of Science, Bangalore, India.

IEEE Log Number 9402823. 
is not, in general, a finite step iterative method. Later, Catedra [16] applied the conjugate gradient method and FFT to compute the charge density over flat plates. This method suffers from the disadvantage that for an efficient utilization of the computational complexity of $O(N \log N)$ offered by FFT, a rectangular grid of sampling points is required. This may not be very feasible for a general arbitrary surface.

Therefore, in this paper a study has been made of the application of a more generalized implementation of CGM for the computation of charge distribution over arbitrary shaped surfaces. The salient features of the work presented in this paper are the following:

- the use of a "natural" polygonal patch modeling for the surfaces, illustrated through examples,

- introduction of a least squares discretisation (LSD) for the computation of the pertinent integral operator and a further simplification on this for faster computation,

- a matrix interpretation of the present implementation leading to certain important conclusions illustrating the differences from an equivalent method of moments formulation,

- and a systematic study of the performance of the two implementations for canonical problems and complex structures.

The organization of the paper is as follows. In Section II the mathematical formulation is presented. The Conjugate Gradient Method (CGM) is briefly discussed in Section III. Section IV shows the equivalence of the present Direct Conjugate Gradient method (DCG) implementation to that of a conventional method of moments solution and the numerical results are presented in Section $\mathrm{V}$.

\section{MATHEMATICAL Formulation}

Let

$$
S_{1}, S_{2}, \cdots, S_{M}
$$

denote the surfaces of $M$ perfectly conducting objects charged to potentials $\phi_{1}, \phi_{2}, \cdots, \phi_{M}$ respectively.

Let

$$
\begin{aligned}
S & =\bigcup_{i=1}^{M} S_{i}(1) \\
\text { and } \quad \phi_{0} & =\phi_{i}, \text { if } \overrightarrow{\mathbf{r}} \in S_{i}, i=1,2, \cdots, M
\end{aligned}
$$

where $\overrightarrow{\mathbf{r}}$ is the position vector with respect to some arbitrary origin The problem is to compute the charge distribution $\sigma$ on $\mathrm{S}$.

When $\overrightarrow{\mathbf{r}} \in S$ we have $[1,5]$,

$$
\phi_{0}(\overrightarrow{\mathbf{r}})=\frac{1}{4 \pi \epsilon} \int_{S} \sigma(\overrightarrow{\mathbf{r}} \prime) G(\overrightarrow{\mathbf{r}}, \overrightarrow{\mathbf{r}} \prime) d S, \overrightarrow{\mathbf{r}} \in S
$$

where $\epsilon$ is the permittivity of free space and $G(\overrightarrow{\mathbf{r}}, \overrightarrow{\mathbf{r}} \prime)=\|\overrightarrow{\mathbf{r}}-\overrightarrow{\mathbf{r}} \prime\|^{-1}$ is the free space Green's function. Equation (3) is a Fredholm integral equation of first kind. The unknown charge distribution can be obtained by solving (3) iteratively.

Then (3) can be written in the operator form as

$$
\hbar \sigma=\phi_{0}
$$

where

$$
K x=\frac{1}{4 \pi \epsilon} \int_{S} x\left(\overrightarrow{\mathbf{r}}^{\prime}\right) G(\overrightarrow{\mathbf{r}}, \overrightarrow{\mathbf{r}} \prime) d S, \overrightarrow{\mathbf{r}} \in S .
$$

Further, the inner product between two real functions $f, g \in L_{2}(S)$ is defined as $[1,2]$,

$$
<f ; g>=\int_{S} f(\overrightarrow{\mathbf{r}}) g(\overrightarrow{\mathbf{r}}) d S
$$

and the norm of a function $f$ as

$$
\|f\|=+\sqrt{\langle f ; g\rangle} \text {. }
$$

With respect to this inner product, it can be shown that the operator $A$ is self adjoint and positive definite [2].

For a numerical implementation, the conductor surface is modeled using polygonal patches. The choice of polygonal patches is motivated by the fact that in most cases, one can find certain "natural" polygons such as triangles or rectangles to discretise a part or parts of surface $S$. In view of this advantage, the polygonal patches are used in this paper and is illustrated through two examples. Further, in what follows, a surface $S$ modeled by patches will be denoted by $S_{N}$ where $N$ is the number of patches.

Thus we have

$$
S \approx S_{N}=\bigcup_{i=1}^{N} P_{i}
$$

where $P_{i}$ is the $i^{\text {th }}$ patch.

With the conducting surface modeled by polygonal panels, if $f$ : $S \rightarrow R$, i.e., a real function defined on $S$, it is approximated by a function $f^{N}: S_{N} \rightarrow R$ defined as

$$
f(\overrightarrow{\mathbf{r}}) \approx f^{N}(\overrightarrow{\mathbf{r}})=\sum_{i=1}^{N} f_{i} \chi_{P_{i}}(\overrightarrow{\mathbf{r}})
$$

where

$$
\chi_{P_{i}}(\overrightarrow{\mathbf{r}})= \begin{cases}1, & \text { if } \overrightarrow{\mathbf{r}} \in P_{i} \\ 0, & \text { elsewhere. }\end{cases}
$$

The coefficients $f_{i}^{N}$ are determined by minimising the quadratic functional $R_{f}$ defined as

$$
R_{f}=\left\|f-f^{N}\right\|
$$

It can be easily shown that $R_{f}$ is minimized if

$$
f_{i}=\frac{\left\langle f ; \chi_{P_{i}}>\right.}{\left\|\chi_{P_{i}}\right\|^{2}} .
$$

Since this approximation minimizes the residual $R_{f}$ in a mean square sense, it is referred to as Least Square Discretisation (LSD) in the rest of the paper.

Further, if $f \in \mathcal{D}(K)$, the domain of $K$, and $g=K f$, then the coefficients of $g^{N}=K f^{N} g_{i}^{N}$ are given by

$$
g_{i}^{N}=\sum_{j=1}^{N} f_{j}^{N} \frac{<K \chi P_{j} ; \chi P_{i}>}{\left\|\chi P_{i}\right\|^{2}} .
$$

It follows that, with these approximations made, the original oprerator (4) reduces to the approximate equation given by

$$
K \sigma^{N}=\phi^{N}
$$

where $\sigma^{N}$ and $\phi^{N}$ are finite dimensional least square approximations to $\sigma$ and $\phi$ respectively.

It may be noted that, in the solution process (see section 3 ), the approximate operator has to be applied to a function in every iteration. The Least Squares Discretisation (LSD) described in (13) involves the computation of a quadruple integral and this requires large amount of CPU time. In order to reduce the CPU time, the second integral in the computation of $\left\langle K \chi_{P_{j}} ; \chi_{P_{i}}\right\rangle$ in (13) has been approximated with a midpoint quadrature rule, i.e.,

$$
<\hbar \chi P_{j} ; \chi P_{i}>=\delta S_{i} \int_{s} \chi_{P_{j}} G\left(\overrightarrow{\mathbf{r}}_{i}, \overrightarrow{\mathbf{r}}^{\prime}\right) d S
$$

where $\delta S_{i}$ is the area of the $i$ th patch and $\overrightarrow{\mathbf{r}}_{i}$ the center of the $i$ th patch. This approximation has been referred to as Point Matching Discretisation $(P M D)$. 
In Section IV the equivalence of the least square approximation to a moment method discretisation of the operator has been derived. In the next section the Classical Conjugate Gradient Algorithm as applied to an operator equation in a Hilbert Space is briefly discussed.

\section{The Conjugate Gradient Algorithm}

The Conjugate Gradient Method (CGM) is an algorithm for the iterative solution of an operator equation of the form

$$
A x=b
$$

set in a Hilbert Space. If $A$ is positive definite, the classical CGM converges to the exact solution in at most $N$ steps in an $N$ dimensional space, and it proceeds as follows [17]:

$$
\begin{aligned}
& x_{0}: \text { arbitrary } \\
& r_{1}=p_{1}=b-A x_{0} \\
& \text { for } n=2,3, \cdots, \text { until convergence } \\
& a_{n}=\frac{\left\|r_{n}\right\|^{2}}{\left\langle p_{n} ; A p_{n}\right\rangle} \\
& x_{n+1}=x_{n}+a_{n} p_{n} \\
& r_{n+1}=r_{n}-a_{n} A p_{n} \\
& b_{n}=\frac{\left\|r_{n}+1\right\|^{2}}{\left\|r_{n}\right\|^{2}} \\
& p_{n+1}=r_{n+1}+b_{n} p_{n}
\end{aligned}
$$

It has been shown [17] that sequence of solutions $\left\{x_{n}\right\}$ generated by this algorithm minimizes the error function

$$
F(x)=<h-x ; A(h-x)>
$$

where $h$ is the exact solution.

However, in an infinite dimensional space, while implementing the above algorithm one has to approximate $b, A x_{0}, p_{n}, r_{n}$ and $A p_{n}$. In such a case the convergence of the solution depends on the approximations made in the numerical implementation [14].

In the present problem, all these approximations have been done as described in Section II. The iterations are terminated if $\left\|r_{n}\right\|^{2} /\|b\|^{2} \leq \delta, \delta>0$.

In the next section, the above Direct Conjugate Gradient scheme (DCG) has been analyzed in the light of the method of moments formalism and the conditions for the two solutions to be identical have also been given.

\section{Equivalent Matrix Problem}

It has been convincingly argued in [14] that because of the limitations in implementing CGM on a computer, one is solving essentially a matrix equation. In the light of this, in this section an attempt has been made is to derive an equivalent matrix formulation and to compare the present implementation with the conventional method of moment formulation. In order to do this, the following notations are introduced.

Let $\mathcal{F}_{\chi}=\operatorname{Span}\left(\chi_{P_{i}} ; i=1,2, \cdots, N\right)$ and let $R^{N}$ denote the $\mathrm{N}$-dimensional real Euclidian space.

Let $\mathcal{B}_{\mathcal{F}}=\left\{\chi_{P_{i}}\right\}_{i=1}^{N}$. It is clear that $\mathcal{B}_{\mathcal{F}}$ forms a basis for $\mathcal{F}_{\chi}$ and hence it follows that $\mathcal{F}_{\chi}$ and $R^{N}$ are isomorphic. Now, if $f \in \mathcal{F}_{\chi}$, let $\bar{f}$ denote the $N$-dimensional vector formed by the coordinates of $f$ with respect to $\mathcal{B}_{\mathcal{F}}$. Obviously $\bar{f} \in R^{N}$.

Restricting the definition of inner product to $\mathcal{F}_{\chi}$, if $f, g \in \mathcal{F}_{\chi}$, we have

$$
<f ; g>=\sum_{i=1}^{N} f_{i} g_{i}\left\|\chi_{P_{i}}\right\|^{2}
$$

and hence,

$$
\|f\|^{2}=\sum_{i=1}^{N} f_{i}^{2}\left\|\chi_{P_{i}}\right\|^{2} .
$$

The Euclidian inner product between two vectors $\bar{x}, \bar{y} \in R^{N}$ is defined as

$$
<\bar{x} ; \bar{y}>_{E}=\bar{x}^{T} \bar{y}
$$

where the superscript $\mathrm{T}$ denotes the transpose.

Let

$$
P=\operatorname{diag}\left(\left\|\chi_{P_{i}}\right\|^{-1}\right), i=1,2, \cdots, N .
$$

Then it can be easily verified that, if $f, g \in \mathcal{F}_{\chi}$, then

$$
\left.<f ; g>=<P^{-1} f ; P^{-1} g\right\rangle_{E} .
$$

Further, define an $N \times N$ matrix $[K]_{\mathcal{B}_{\mathcal{F}}}$ by

$$
\begin{aligned}
{[K]_{B_{\mathcal{F}}} } & =\left[K_{i j}\right], \\
\mid \text { where } K_{i j} & =\frac{\left\langle K \chi_{P_{j}} ; \chi_{P_{i}}\right\rangle}{\left\|\chi_{P_{i}}\right\|^{2}} .
\end{aligned}
$$

Now, if $f \in \mathcal{F}_{\chi}$ and $g=K f$ then it follows from (12) and (13) that

$$
\bar{g}=[K]_{\mathcal{B}_{\mathcal{F}}} \bar{f}
$$

and we refer to $[K]_{B_{\mathcal{F}}}$ as the matrix representation of the operator $\mathrm{K}$ defined in section (2) with respect to $\mathcal{B}_{\mathcal{F}}$.

It may be noted that this matrix equation can be derived by choosing the basis functions to be $\left\{\chi_{P_{i}}\right\}$ and the weight functions $\left\{\chi_{P_{i}} /\left\|\chi_{P_{i}}\right\|^{2}\right\}$. However, unless $\left\|\chi_{P_{i}}\right\|=\left\|\chi_{P_{j}}\right\|$ for all $i, j$, the matrix $[K]_{\mathcal{B}_{\mathcal{F}}}$ will not be symmetric. Hence, for the matrix equation (26) the classical CGM is not defined. Since the matrix is unsymmetric, an appropriate preconditioning technique has to be used. One such approach to this is to apply the CGM to the normalized form of (23) [11]. This has the disadvantage that the condition number of the matrix is squared. In addition to this, the application of CGM requires two matrix vector products, which can be expensive, particularly when the elements are computed "on the fly" [14].

Therefore, the foregoing discussion suggests that the present implementation of DCG introduces some preconditioning into (26) for retaining the self-adjointness property of the original operator equation defined in (4). This implicit preconditioning is derived next.

For this, (26) is rewritten as

$$
P^{-1}[K]_{\mathcal{B}_{\mathcal{F}}} \bar{f}=P^{-1} \bar{g}
$$

where $P^{-1}$ is the inverse of $P$ defined in (22). Now, define

$$
\begin{aligned}
& \widehat{[K}_{\mathcal{B}_{\mathcal{F}}}=P^{-1}[K]_{\mathcal{B}_{\mathcal{F}}} P \\
& \left.\begin{array}{l}
\bar{f} \prime=P^{-1} \bar{f} \\
\bar{g} \prime=P^{-1} \bar{g}
\end{array}\right\} \\
& {\widehat{[K]_{\mathcal{B}}}}_{\mathcal{f}^{\prime}}=\bar{g} \prime
\end{aligned}
$$

It can be easily verified that $\widehat{[K}_{\mathcal{B}_{\mathcal{F}}}$ is symmetric and positive definite and a direct substitution of $(28)$ and (29) into (30) results in (27). Hence, it follows that the classical CGM can be applied for the solution of (30), though not for (26). Further, from (23), (26), (28), and (29) it is clear that the solution obtained by solving (30) by CGM is the same as the one by DCG described in Sections III and II.

Therefore, it follows that the present implementation of DCG retains the symmetry of the operator to the matrix equation through an implicit similarity transformation, which, however, does not alter 


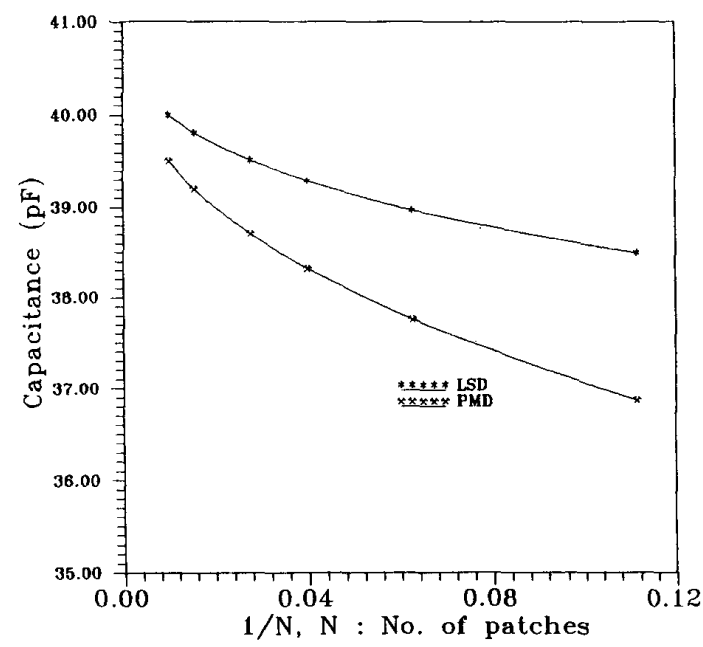

Fig. 1. Plot of capacitance versus $1 / N$ for the square plate of the unit side.

the eigen values of the matrix. Hence, if the preconditioning used for solving (26) is different from the similarity transformation inherent in the DCG, the numerical results could be different.

Further, it follows that the Point Matching Discretisation (PMD), is equivalent to a method of moments formulation with pulse expansion and point matching. Hence, from the discussion it is clear that when all the patches have the same area, the PMD solution would be identical to that given by the MoM. This is substantiated by the numerical computation of the capacitance of a square plate presented in Section $\mathrm{V}$.

\section{ILLUSTRATIVE EXAMPLES}

In this section, results have been presented for the computation of the capacitance and charge distribution on arbitrarily shaped conductors. In order to validate the formulation with the results available in the literature, canonical shapes such as rectangular plates, and circular discs have been considered. The ability of the techniques presented in this paper to handle statics problems involving arbitrary shapes has been demonstrated by computing the capacitance of a pyramid with square base mounted on top of a cube. All the computations reported in this paper have been carried out on a Control Data CD4360, which has an R3000 MIPS processor running at 33 MHz.

Harrington [1] has used the method of moments formulation with pulse basis and point matching and has shown that the capacitance of a square plate of $1 \mathrm{~m}$ side converges to $40 \mathrm{pF}$ and this value has has been used for gauging the convergence of the algorithms presented in this paper. Both LSD and PMD have been applied. The plate has been modeled with square patches. The number of patches has been varied from 9 to 100 in order to study the convergence of the results.

Fig. 1 shows the computed capacitance of a square plate as a function of the inverse of the number of square patches. It can be seen that the values obtained by PMD are identical to that obtained by Harrington [1]. This is as predicted by the analysis of Section IV and (15). The percentage error has been calculated as the deviation of the computed capacitance from $40 \mathrm{pF}$. This is presented in Fig. 2. For a specific accuracy the number of patches required in the LSD is much smaller, for example to obtain the value $39.5 \mathrm{pF} / \mathrm{m}$, corresponding to an error of $1.0 \%$, PMD requires 100 patches whereas LSD requires only 36 patches. Clearly there

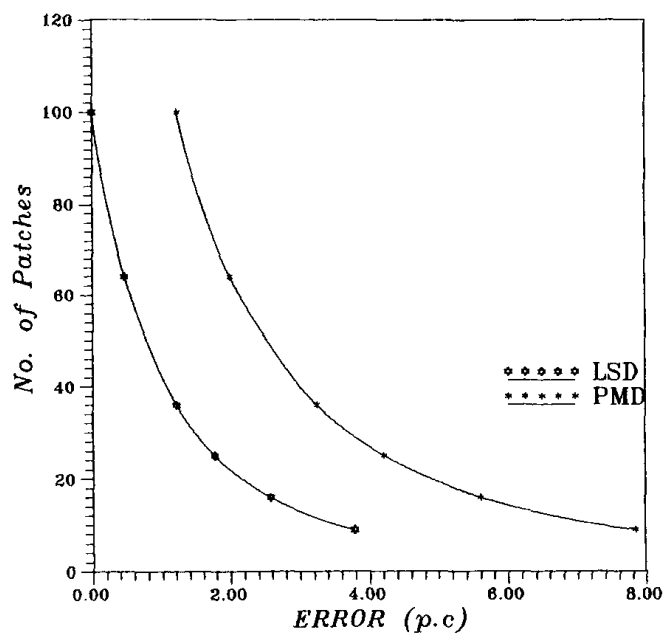

Fig. 2. Plot of the number of patches versus error for the square plate.

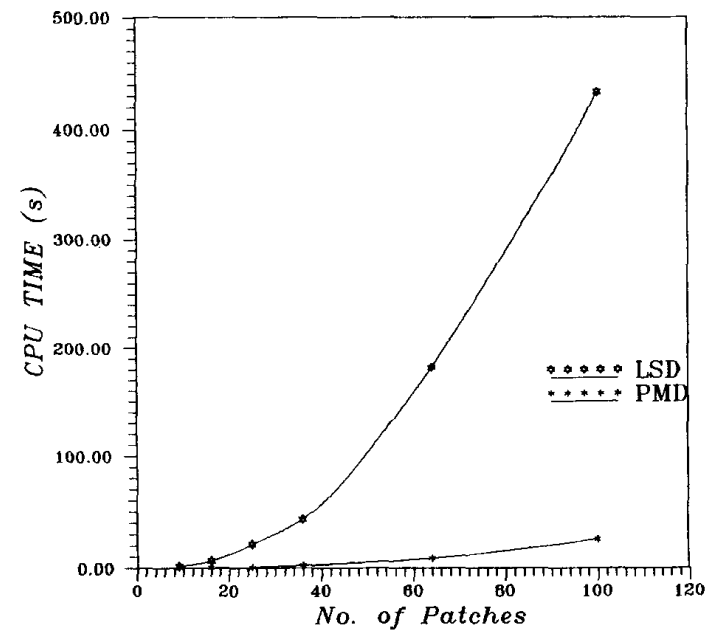

Fig. 3. Plot of CPU time versus number of patches for square plate.

is an advantage for the LSD formulation over the point matching discretisation as far as the number of patches are concerned. However, it is to be anticipated that the LSD would require larger computational effort. To illustrate this, the CPU time required has been depicted in Fig. 3 as a function of the number of patches. Though LSD takes larger CPU time than PMD, the accuracy that it yields is better for the same number of patches-as seen in Fig. 2. For practical applications, the CPU time required for a prespecified accuracy is of concern. This is plotted in Fig. 4. It is clear that for errors greater than $3 \%$, the computer time required is almost equal for both LSD and PMD. Because, the integrals are evaluated more accurately in LSD, it is inherently capable of yielding highly accurate solutions though at the expense of computer resources. Fig. 1 indicates the monotonic convergence in the capapcitance obtained with the present formulation also.

As a second example, a circular disc is modeled using triangular patches. Rao et al. [3] have analyzed a circular disc using triangular patches and method of moments. They have also quoted an analytical 


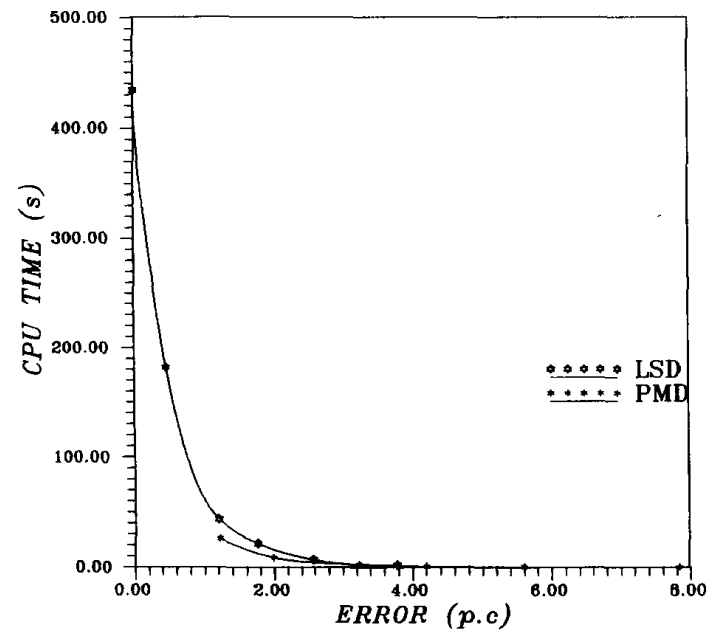

Fig. 4 Plot of error (\%) versus CPU time for the square plate problem.

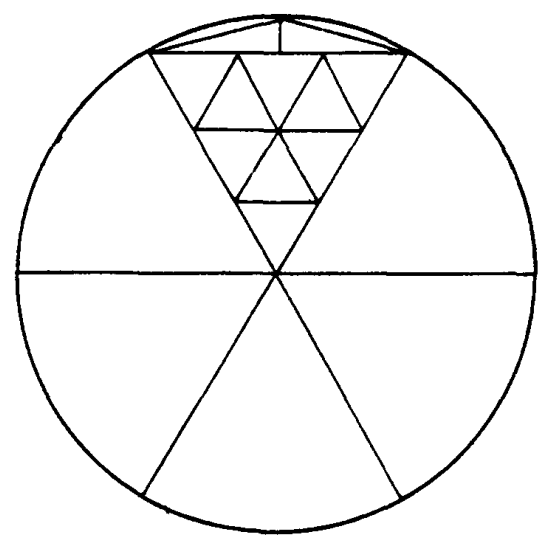

Fig. 5. Triangular patch model of unit disc with $N_{\theta}=6, N_{S}=11$, and $N_{P}=66$.

expression for its capacitance, from which the capacitance of a disc of unit radius $(1 \mathrm{~m})$ can be obtained as $70.83 \mathrm{pF}$. A typical model of the disc is shown in Fig. 5, wherein the $N_{\theta}$ is used to denote the sectors into which the disc has been divided. Two values of $N_{\theta}=6$ and 12 are taken in modeling the circular disc. The number of triangular patches in each sector $\left(N_{S}\right)$ and hence the total number of patches ( $N_{P}=N_{\theta} \cdot N_{S}$ ) are varied. The capacitance of the disc as a function of the number of patches $\left(N_{P}\right)$, for $N_{\theta}=6$ and $N_{\theta}=12$ is plotted in Fig. 6. The capacitance obtained when $N_{\theta}=12$ is closer to the exact value of $70.83 \mathrm{pF}$. As in the case of the square plate the LSD yields solutions that are more accurate for both $N_{\theta}=6$ and $N_{\theta}=12$. When the disc is divided into 12 sectors $\left(N_{\theta}=12\right)$ and each sector is modeled with 6 triangular patches $\left(N_{P}=72\right)$, the capacitance obtained is seen from Fig. 6 as $69.90 \mathrm{pF}$ for LSD. For a choice of six sectors and 24 triangular patches in each sector $\left(N_{P}=162\right)$ the capacitance is seen to be $68.32 \mathrm{pF}$. Thus it is clear that the choice of $N_{\theta}=12$ yields more accurate results than when $N_{\theta}=6$. This suggests that, in addition to the number of patches their distribution also affects in the rate of convergence. In order to compare the accuray obtained in the charge density, it is plotted as a function of radial distance in Fig. 7. The circular disc has been

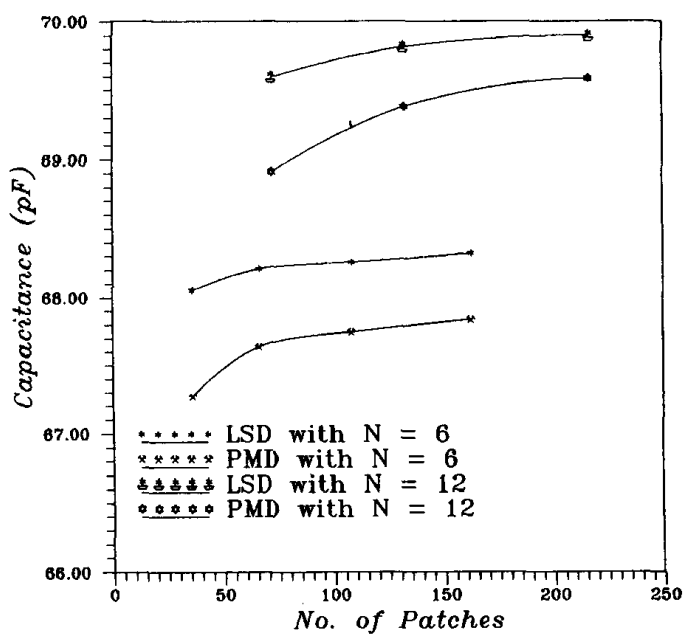

Fig. 6. Plot of capacitance versus number of patches for a circular disc of unit radius.

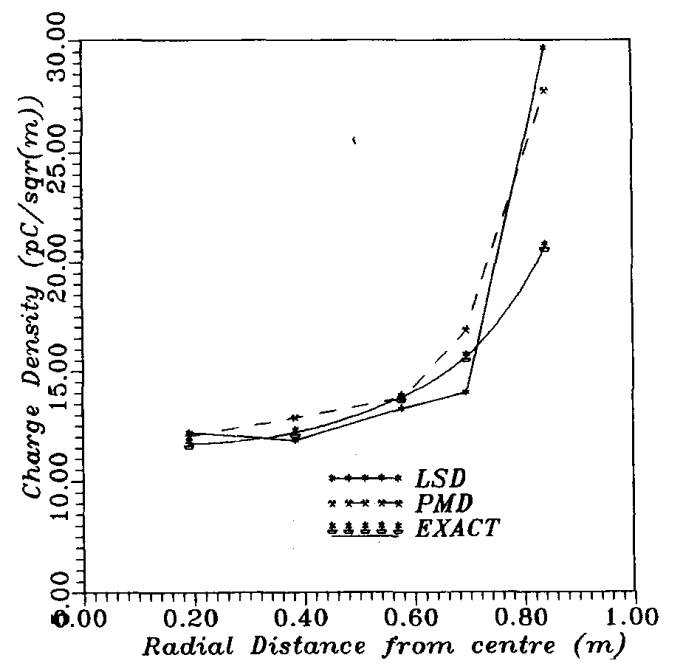

Fig. 7. Plot of charge density as a function of radial distance for the circular disc.

modeled with $N_{\theta}=6$ and $N_{P}=66$. The exact value has been computed using the expression [3]

$$
\sigma(\rho)=\frac{4 \epsilon_{0}}{\pi \sqrt{1-\rho^{2}}}
$$

with one volt excitation. It can be seen that PMD is closer to the exact solution than LSD. This is because LSD minimizes the error in $\sigma$ in a "mean square" sense, whereas PMD is a collocation approach.

The above examples demonstrate that the capacitance converges monotonically for both LSD and PMD. Further, the LSD requires lesser number of patches for the same accuracy levels than PMD, and is inherently more accurate albeit demanding more computer resources.

In order to illustrate the capability of the polygonal patch modeling, the next example considered is that of a pyramid with square base mounted on a cube. The pyramid is taken to be of unit height with a square base of unit side. The triangular faces were modeled using triangular patches and the square faces with square patches. It can 


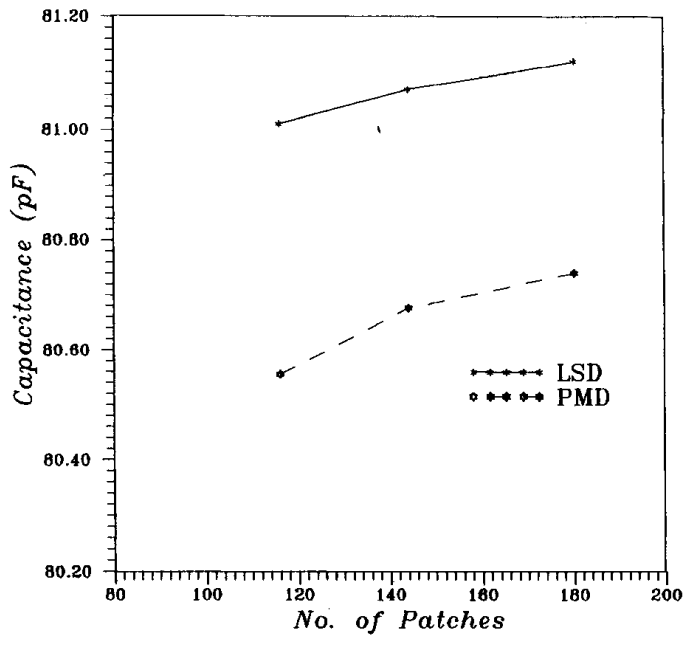

Fig. 8. Capacitance of pyramid mounted on a cube versus number of patches.

be seen from Fig. 8 that the capacitance converges monotonically to $81.15 \mathrm{pF}$.

\section{Conclusions}

A study has been made on the application of conjugate gradient method for statics problems involving arbitrary shaped conductors. The versatility of the method has been demonstrated by calculating the capacitance of simple as well as complex geometrical shapes. Polygons that are most general to the shape of the arbitrary conductor geometry have been chosen for modeling. In all cases the technique converges to the solution monotonically and in finite steps.

Two variations referred to as LSD and PMD have been proposed for evaluating the integrals involved in the CGM formulation. It has been shown that LSD, in all cases, requires lesser number of patches, and is inherently capable of yielding more accurate solutions. Whenever the error levels of greater than $3 \%$ can be tolerated, both LSD and PMD take approximately the same amount of computer time. The PMD solutions have been shown both analytically and using numerical examples, to converge to that given by $\mathrm{MoM}$ whenever all the patches have the same area.

\section{ACKNOWLEDGMENT}

The authors thank Dr. M. Dutta, and Reena Sharma for many helpful suggestions, Prof. R. P. Shenoy for his critical review and Rajalakshmi Sampath for help in the preparation of the manuscript.

\section{REFERENCES}

[1] R. F. Harrington, "Matrix methods for field problems," Proc. IEEE, vol. 55, pp. 136-149, Feb. 1967.

[2] R. F.Harrington, Field Computation by Moment Methods. New York: MacMillan, 1968

[3] S. M. Rao, A. W. Glisson, D. R. Wilton, and B. Sarma Vidula, "A simple numerical solution procedure for statics problems involving arbitrary shaped conductors," IEEE Trans. Antennas Propagat., vol. AP-27, pp. 604-607, Sept. 1979

[4] R. F. Harrington and T. K. Sarkar, "Boundary elements and method of moments," in Boundary Elements: Proc. Fifth Int. Conf., Nov. 1983

[5] G. T. Symm, "Introduction to the application of boundary element method to electrostatics," in Boundary Element Technology, C. A. Brebbia, et al., ed. Wein, Austria: Springer-Verlag, 1986.
[6] D. G. Dudley, "Error minimization and convergence in numerical methods," Electromagnetics, vol. 5, no.2-3, pp. 89-97, 1985.

[7] T. K. Sarkar, "A note on the variational method (Rayleigh-Ritz), Galerkins method and the method of least squares," Radio Science, vol. 18, no. .6, pp. 1207-1224, Nov.-Dec. 1983.

[8] T. K. Sarkar, "A note on the choice of weighting functions in the method of moments," IEEE Trans. Antennas Propagat., vol. AP-33, pp. 436-441, Apr. 1985.

[9] T. K. Sarkar, A. R. Djordevic, and E. Arvas, "On the choice of expansion and weighting functions in the numerical solution of operator equations," IEEE Trans. Antennas Propagat., vol. AP-33, pp. 988-996, Sept. 1985.

[10] K. Rektorys, Variational Methods in Mathematics, Science, and Engineering. D. Reidel Publishing Co., 1972.

[11] T. K. Sarkar and S. M. Rao, "The application of the conjugate gradient method for the solution of electromagnetic scattering from arbitrarily oriented wire antennas," IEEE Trans. Antennas Propagat., vol. AP-32, pp. 398-404, Apr. 1984.

[12] P. M. Vanden Berg, "Iterative computational techniques in scattering based upon the integrated square error criterion," IEEE Trans. Antennas Propagat., vol. AP-32, pp. 1063-1071, Oct. 1984.

[13] T. K. Sarkar and E. Arvas, "On a class of finite step iterative methods (conjugate directions) for the solution of an operator equation arising in electromagnetics,"IEEE Trans. Antennas Propagat., vol. AP-33, pp. 1058-1066, Oct 1985.

[14] S. L. Ray and A. F. Peterson, "Error and convergence in numerical implementation of the conjugate gradient method," IEEE Trans. Antennas Propagat., vol. AP-36, pp.1824-1827, 1986.

[15] T. K. Sarkar and S. M. Rao, "An iterative method for solving electrostatic problems," IEEE Trans. Antennas Propagat., vol. AP-30, pp. 611-616, July 1982

[16] M. F. Catedra, "Solution to some electromagnetic problems using fast Fourier transform with conjugate gradient method," Electron. Lett., vol. 22, no. 20, pp. 1049-1051, Sept. 25, 1986.

[17] R. M. Hayes, "Iterative methods for solving linear problems on Hilbert space," U. S. Dept. of Commerce, Nat. Bureau of Standards, Applied Mathematics Series-39, pp. 71-103, Sept. 1955.

\section{A Radar Target Discrimination Scheme Using the Discrete Wavelet Transform for Reduced Data Storage}

\author{
E. J. Rothwell, Senior Member, IEEE, K. M. Chen, Fellow, IEEE, \\ D. P. Nyquist, Senior, Member, IEEE, J. E. Ross, Member, IEEE, \\ and R. Bebermeyer, Student Member, IEEE
}

\begin{abstract}
A correlative radar target discrimination scheme using the transient scattered-field response is proposed. This scheme uses a onedimensional discrete wavelet transform on the temporal response to reduce the amount of data that must be stored for each anticipated aspect angle. Experimental results show that a reduction in stored data of sixteen to one still allows accurate discrimination in adverse noise situations with signal-to-noise ratios as low as $-5 \mathrm{~dB}$.
\end{abstract}

\section{INTRODUCTION}

A fascinating variety of radar target discrimination schemes have been proposed in the past several years. Each of these techniques must deal with the complicated dependence of scattered

Manuscript received August 23, 1993; revised January 31, 1994. This work was supported by the ThermoTrex Coproration under Purchase Order 22068, and in part by the Northeast Consortium for Engineering Education under Purchase Order NCEE/A303/23-93 and the Office of Naval Research under Grant N00014-93-1-1272.

The authors are with the Department of Electrical Engineering, Michigan State University, East Lansing, MI 48824 USA.

IEEE Log Number 9402826. 